\title{
Efficient Modeling of Antennas with Finite Conductivity using Calderón Preconditioning
}

\author{
Michiel Gossye, Dries Vande Ginste, Daniël De Zutter, and Hendrik Rogier \\ IDLab/Electromagnetics Group, Department of Information Technology \\ Ghent University/imec, Belgium \\ Michiel.Gossye@UGent.be
}

\begin{abstract}
A Calderón preconditioner for a novel single-source integral equation, which allows the simulation of high dielectric contrasts and lossy conductors, is introduced. It is shown that this preconditioner avoids breakdown when simulating scattering problems including high permittivities. This is corroborated by a numerical example of a receiving copper dipole antenna.
\end{abstract}

\section{INTRODUCTION}

In recent research, Calderón preconditioners (CPs) were developed to resolve dense mesh breakdown problems that occur when solving boundary integral equations (BIEs). This preconditioner is based on the self-regularization effect of the electric field integral operator (EFIO) [1]. In [2], such a preconditioner was developed for the Poggio-Miller-Chang-Harrington-WuTsai (PMCHWT) equation, resolving the dense mesh breakdown of this operator. However, for high dielectric contrasts, this preconditioner is not able to stabilize the system matrix [3]. Therefore, the accurate simulation of high contrasts including lossy conductors still remains an active research topic. In this contribution, a Calderón preconditioner for a novel single-source equation is introduced that resolves dense mesh breakdown at high dielectric contrasts. In Section II, the theoretical framework and spectral analysis of this method are described. These results are then validated by a numerical example of a lossy dipole antenna in Section III.

\section{THEORY}

Assume a homogeneous object (characterized by $\varepsilon^{\prime}, \mu^{\prime}$ and $k^{\prime}$ ), embedded in a homogeneous background medium (characterized by $\varepsilon, \mu$ and $k$ ), as shown in Fig. 1 (a). An incident time-harmonic ( $e^{j \omega t}$ time dependence) electromagnetic field $\left(\mathbf{E}^{i n}, \mathbf{H}^{i n}\right)$ impinges on this object, generating a scattered field $\left(\mathbf{E}^{s c}, \mathbf{H}^{s c}\right)$. Consider next the equivalent situation in Fig. 1 (b), where the material inside the object is replaced by that of the external region. Furthermore, a magnetic virtual current $\mathbf{m}$ residing on the boundary of the object is introduced. This current, which is to be determined, must generate the same scattered fields as in the original problem. After introduction of the Poincaré-Steklov operator $\mathcal{P}$, satisfying the property $\mathcal{P}(-\hat{\boldsymbol{n}} \times \boldsymbol{e})=\hat{\boldsymbol{n}} \times \boldsymbol{h}$, and by imposing continuity of the electromagnetic fields on the object boundary $\Gamma$, we find the following single-source matrix equation:

$$
\left[\begin{array}{cc}
\mathcal{K}+\frac{1}{2} & -1 \\
\frac{\mathcal{T}}{\eta} & -\mathcal{P}
\end{array}\right] \cdot\left[\begin{array}{c}
\boldsymbol{m} \\
-\boldsymbol{u}_{n} \times \boldsymbol{E}
\end{array}\right]=\left[\begin{array}{c}
\boldsymbol{u}_{n} \times \boldsymbol{E}^{i n} \\
-\boldsymbol{u}_{\boldsymbol{n}} \times \boldsymbol{H}^{i n}
\end{array}\right],
$$

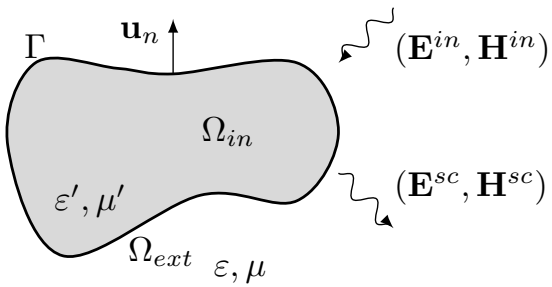

(a) Original problem

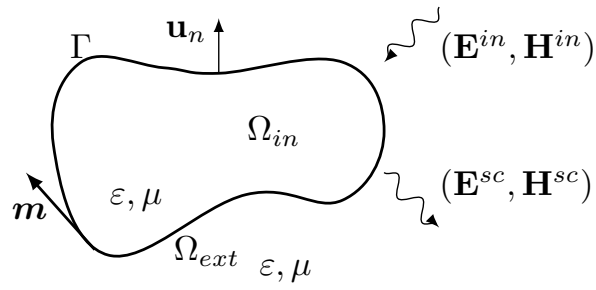

(b) Equivalent problem

Fig. 1. In (a), a homogeneous object $\Omega_{\text {in }}$ is embedded in a homogeneous background medium $\Omega_{e x t}$. Interaction of the incident field $\left(\boldsymbol{E}^{i n}, \boldsymbol{H}^{i n}\right)$ results in a scattered field $\left(\boldsymbol{E}^{s c}, \boldsymbol{H}^{s c}\right)$. In (b), the object is filled with the medium of $\Omega_{e x t}$ and a surface current density $\mathbf{m}$, generating $\left(\boldsymbol{E}^{s c}, \boldsymbol{H}^{s c}\right)$, is introduced on $\Gamma$.

with $\mathcal{T}$ and $\mathcal{K}$ the electric and magnetic field integral operator of $\Omega_{\text {ext }}$ [2], $\eta=\sqrt{\mu / \varepsilon}$ the impedance of $\Omega_{e x t}$, and $\boldsymbol{u}_{n} \times \boldsymbol{E}$ the total tangential electric field on the boundary. Next, we left multiply (1) with the following Calderón preconditioner:

$$
\left[\begin{array}{cc}
1 & 0 \\
0 & -\eta^{\prime} \mathcal{T}^{\prime}
\end{array}\right]
$$

with $\eta^{\prime}$ and $\mathcal{T}^{\prime}$ the impedance and the EFIO of $\Omega_{\text {int }}$, respectively. This results, after discretization, in:

$$
\begin{gathered}
{\left[\begin{array}{cc}
K+\frac{1}{2} G & -G \\
-\frac{\eta^{\prime}}{\eta} T^{\prime} \cdot\left(-G^{T}\right)^{-1} \cdot T & K^{\prime}+\frac{1}{2} G
\end{array}\right] \cdot\left[\begin{array}{c}
\boldsymbol{M} \\
\boldsymbol{E}_{t}
\end{array}\right]} \\
=\left[\begin{array}{c}
\boldsymbol{E}_{t}^{i} \\
\eta^{\prime} T^{\prime}\left(-G^{T}\right)^{-1} \boldsymbol{H}_{t}^{i}
\end{array}\right] .
\end{gathered}
$$

The vectors $\boldsymbol{M}$ and $\boldsymbol{E}_{\boldsymbol{t}}$ in (3) collect the coefficients after expanding $\boldsymbol{m}$ and $-\boldsymbol{u}_{n} \times \boldsymbol{E}$ in Rao-Wilton-Glisson (RWG) functions. Furthermore, $T$ and $\boldsymbol{H}_{t}^{i}$ are tested with rotated RWG basis functions, while $K, K^{\prime}, \boldsymbol{E}_{t}^{i}$ and the Gramian matrix $G$ are tested with rotated Buffa-Christiansen (BC) [4] functions. $T^{\prime}$ is expanded and tested with BC and rotated BC functions, respectively. 


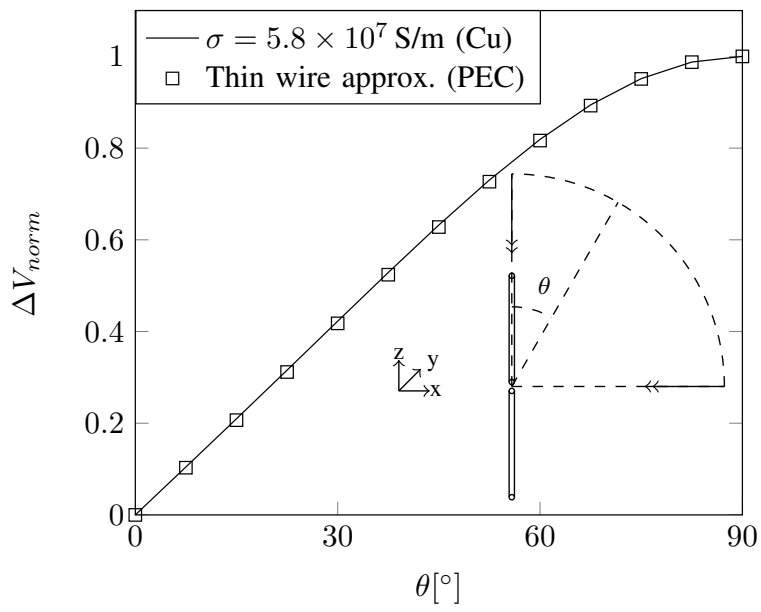

Fig. 2. Normalized voltage difference between the terminals of a copper dipole antenna with axis along the $\mathrm{z}$-axis, at a frequency of $100 \mathrm{MHz}$. The antenna has a total length of $\lambda / 2$, a radius of $\lambda / 100$, a terminal gap of $\lambda / 150$ and 2552 mesh elements. The incident wave is given by $\boldsymbol{E}^{i n}=$ $\left(\cos (\theta) \boldsymbol{u}_{x}-\sin (\theta) \boldsymbol{u}_{z}\right) e^{j k(\sin (\theta) x+\cos (\theta) z)}$ (as depicted in the schematic inset). The data are compared to the analytical approximation of a perfectly conducting thin wire antenna.

The convergence time of the iterative solution of (3) depends on the eigenvalue distribution of the system matrix [5]. It can be proven [6] that the eigenvalue accumulation points of the system matrix in (3) are given by:

$$
\lambda_{1, \pm}=\frac{1}{2} \pm \frac{1}{2} \sqrt{\frac{\varepsilon}{\varepsilon^{\prime}}} j
$$

and

$$
\lambda_{2, \pm}=\frac{1}{2} \pm \frac{1}{2} \sqrt{\frac{\mu^{\prime}}{\mu}} j .
$$

If non-magnetic materials are assumed, one observes that the accumulation points only tend to zero or infinity when $\varepsilon \rightarrow 0$ or $\varepsilon \rightarrow-1$. Hence, in most practical engineering situations such as antenna design, which includes modeling of high dielectric contrast and conductive media, this formulation is well-conditioned.

\section{EXAMPLE}

As a numerical validation of the formulation in (3), we consider a copper dipole antenna at a frequency of $100 \mathrm{MHz}$ (inset of Fig. 2). The antenna has a total length of $\lambda / 2$ and a radius of $\lambda / 100$. The gap between the rods equals $\lambda / 150$. In Fig. 2 , the simulated voltage difference between the terminals is shown as a function of the angle of incidence of the incoming plane wave. This result is validated by the analytical thin wire approximation. The open-circuit voltage of the copper dipole shows a root mean square (rms) difference of $0.3 \%$ compared to this reference solution.

In Fig. 3, the number of iterations until convergence (rms error $<10^{-10}$ ) and the condition number of the system matrix are given as a function of the number of unknowns for both the method proposed in this paper and for the CP-PMCHWT equation. On the one hand, it can be seen that the number

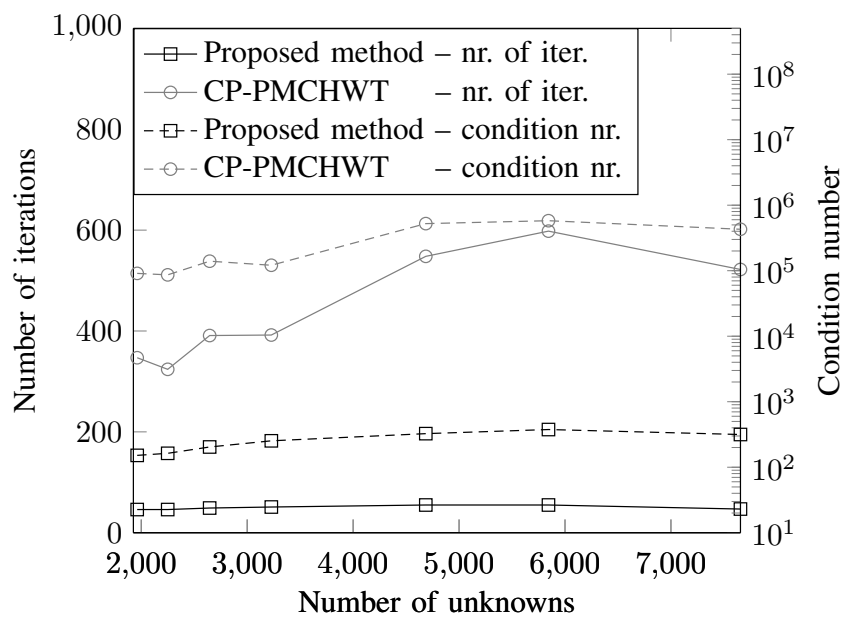

Fig. 3. Iterations until convergence (left y-axis) and condition number (right $y$-axis) as a function of the number of unknowns for the copper dipole antenna of Fig. 2 for an incident field $\boldsymbol{E}^{i n}=\boldsymbol{u}_{x} e^{j k z}$, for the proposed method and for the CP-PMCHWT equation.

of iterations $(\approx 50)$ and the condition number $(\approx 300)$ of the proposed method remain at a stable low value for an increasing number of unknowns, validating the claim that dense mesh breakdown does not occur. The CP-PMCHWT method on the other hand suffers from ill-conditioning, resulting in longer convergence times.

\section{CONCLUSION}

In this paper, a Calderón preconditioner for a novel singlesource method that resolves the breakdown problem at high dielectric contrasts is presented. A receiving, copper dipole antenna is considered. The results demonstrate the practical applicability of the proposed method to antenna design and other scattering problems.

\section{ACKNOWLEDGMENT}

The authors thank the Research Foundation Flanders (FWO) for supporting this research.

\section{REFERENCES}

[1] F. P. Andriulli, K. Cools, H. Bağci, F. Olyslager, A. Buffa, S. Christiansen and E. Michielssen, "A multiplicative Calderón preconditioner for the Electric Field Integral Equation," IEEE Trans. Antennas Propag., vol. 56, no. 8, pp. 2398-2412, Aug. 2008.

[2] K. Cools, F. P. Andriulli and E. Michielssen, "A Calderón multiplicative preconditioner for the PMCHWT integral equation," IEEE Trans. Antennas Propag., vol. 59, no. 12, pp. 4579-4587, Dec. 2011.

[3] Pasi Ylä-Oijala and Sami P. Kiminki, "Challenges in developing efficient Calderón preconditioners for resonating or high material contrast penetrable objects," J. Comput. Appl. Math., vol. 289, pp. 296-305, Dec. 2015.

[4] A. Buffa and S. H. Christiansen, "A dual finite element complex on the barycentric refinement," Math. Comput. , vol. 76, no. 260, pp. 1743 1769, 2007.

[5] P. Yla-Oijala and M. Taskinen, "Application of combined field Integral equation for electromagnetic scattering by dielectric and composite objects," IEEE Trans. Antennas Propag., vol. 53, pp. 1168-1173, Mar. 2005.

[6] M. Gossye, M. Huynen, D. Vande Ginste, D. De Zutter and H. Rogier, "A Calderón Preconditioner for High Dielectric Contrast Media", IEEE Trans. Antennas Propag., in press. 